\title{
The Role of Cell-Free Plasma DNA in Patients with Cardiorenal Syndrome Type 1
}

\author{
Grazia Maria Virzi ${ }^{a}$ b Anna Clementic Sabrina Milan Manani ${ }^{a}{ }^{a} b$ \\ Chiara Castellani $^{d}$ Giovanni Giorgio Battaglia $^{c}$ Annalisa Angelini ${ }^{d}$ \\ Giorgio Vescovo ${ }^{e, f}$ Claudio Ronco ${ }^{a, b}$, d
}

aIRRIV-International Renal Research Institute Vicenza, Vicenza, Italy; ${ }^{b}$ Department of Nephrology, Dialysis and Transplant, San Bortolo Hospital, Vicenza, Italy; ${ }^{\circ}$ Department of Nephrology and Dialysis, Santa Marta and Santa

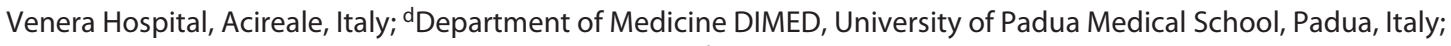

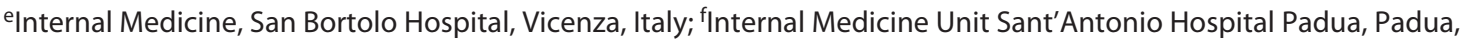
Italy

\section{Keywords}

Cell-free plasma DNA · Cardiorenal syndrome .

Heart failure $\cdot$ Apoptosis $\cdot$ Inflammation

\begin{abstract}
Background: Recent research highlighted the potential role of circulating cell-free DNA (cfDNA), resulted by apoptosis or cell necrosis, as a prognostic marker in the setting of different clinical conditions. Cardiorenal syndrome type 1 (CRS type 1) is characterized by a rapid worsening of cardiac function leading to acute kidney injury (AKI). Apoptosis of renal epithelial cells is proposed as a mechanism involved in CRS type 1. In this study, we investigated cfDNA levels in patients with acute heart failure (AHF) and CRS type 1 and the possible correlation between cfDNA levels and inflammatory and apoptotic parameters. Methods: We enrolled 17 AHF patients and 15 CRS type 1 who exhibited AKI at the time of admission (caused by AHF) or developed AKI during the first $48 \mathrm{~h}$ from admission. cfDNA was extracted from plasma and quantified by real-time polymerase chain reaction. Plasma levels of NGAL, tumor necrosis factor-a, interleukin (IL)-6, IL-
\end{abstract}

(C) 2021 The Author(s)

Published by S. Karger AG, Basel

This is an Open Access article licensed under the Creative Commons Attribution-NonCommercial-4.0 International License (CC BY-NC) (http://www.karger.com/Services/OpenAccessLicense), applicable to the online version of the article only. Usage and distribution for commercial purposes requires written permission.
18, and caspase-3 were measured. Results: We observed significantly higher levels of cfDNA in patients with CRS type 1 than patients with AHF. Caspase-3, IL-6, IL-18, and NGAL levels resulted significantly increased in patients with CRS type 1. Moreover, a positive correlation between cfDNA levels and caspase-3 levels was found, as well as between cfDNA levels and IL- 6 and renal parameters. Conclusion: Our study explores the premise of cfDNA as a marker for apoptosis and inflammation in CRS type 1 patients. cfDNA could potentially serve as an index for noninvasive monitoring of tissue damage and apoptosis in patients with AKI induced by AHF.

(c) 2021 The Author(s).

Published by S. Karger AG, Basel

\section{Introduction}

Cell-free plasma DNA (cfDNA) is circulating extracellular DNA deriving from apoptosis or cell necrosis. Small amounts of cfDNA are normally detected in the blood of healthy individuals, but its levels increase in several clinical conditions, including sepsis [1-3], trauma [4], myo- 
cardial infarction [5], stroke [6], pregnancy-associated disorders [7], hemodialysis and peritoneal dialysis [8], and cancer [9].

Cardiorenal syndrome type 1 (CRS type 1 ) is characterized by a fast falling of cardiac function leading to acute kidney injury (AKI). Reduced renal perfusion due to low cardiac output and neurohormonal activation are typical of CRS type 1, and they are responsible for renal ischemia with AKI. In addition, nonhemodynamic mechanisms (inflammation, immune dysregulation, etc.) are involved in the pathogenesis of CRS type 1. In this context, apoptosis of epithelial cells in proximal and distal tubules has a key role in ischemic renal damage [10]. In higher organisms, apoptosis is mediated by caspases, a family of cysteine proteases cleaving after an aspartate residue in their substrates [11]. cfDNA and its connection with apoptosis in CRS type 1 are not known: its role has never been studied in this condition.

In this study, we compared cfDNA levels between patients with acute heart failure (AHF) and patients with CRS type 1. Moreover, we investigated the possible correlation between cfDNA levels and inflammatory and renal parameters and caspase- 3 in patients with AHF and CRS type 1.

\section{Materials and Methods}

\section{Patients}

Patients with AHF admitted into the Internal Medicine Department of San Bortolo Hospital were screened for the enrollment in the study. Patients with AKI before the episode of AHF or with other possible causes of AKI were excluded. An estimated glomerular filtration rate $(e G F R)<45 \mathrm{~mL} / \mathrm{min} / 1.73 \mathrm{~m}^{2}$ or with a history of kidney transplantation were exclusion criteria. Furthermore, septic patients and hypotensive patients who required inotropic support prior to the diagnosis of AKI were excluded. Furthermore, we excluded patients exposed to contrast media in the $72 \mathrm{~h}$ preceding AKI and patients without baseline level of serum creatinine (SCr). We considered as the baseline value, at least 1 SCr level of the last 3 months before the admission for all patients enrolled in the study. Finally, we enrolled 17 AHF and 15 CRS type 1 patients. 8/15 CRS type 1 patients exhibited AKI at the time of admission (caused by AHF) and 7/15 developed AKI during the course of hospitalization (first $48 \mathrm{~h}$ from admission).

Clinical data, blood pressure, SCr, blood urea, hemoglobin, brain natriuretic peptide were evaluated and collected at admission. AHF was defined by the European Society of Cardiology (ESC) guidelines [12]. AKI was defined by the Acute Kidney Injury Network (AKIN) criteria [13]. CRS type 1 was defined according to the current classification system. SCr was measured with an enzymatic method, isotope dilution mass spectrometry traceable by an automatic analyzer (Dimension Vista; Siemens Healthcare, Tarrytown, NY, USA), and eGFR was calculated with the CKDEPI equation [14].

Cell-Free Plasma DNA in CRS Type 1 Patients

\section{Sample Collection}

EDTA blood samples were collected from all 32 patients within $8 \mathrm{~h}$ from the admission into the Internal Medicine. We also collected blood sample within $24 \mathrm{~h}$ of AKI for patients who developed CRS type 1. For AHF patients, we used the blood sample from the admission; we used the blood sample within $24 \mathrm{~h}$ of AKI for patients who developed CRS type 1 . Samples were subsequently centrifuged for $7 \mathrm{~min}$ at $1,600 \mathrm{~g}$. After centrifugation, plasma was immediately stored at $-80^{\circ} \mathrm{C}$ until the use.

\section{Determination of NGAL Levels}

Quantitative determination of NGAL was performed by an Alere Triage NGAL Panel (Alere, San Diego, CA, USA) at admission or within $24 \mathrm{~h}$ of AKI for patients who developed CRS type 1.

\section{Isolation and cfDNA Extraction}

cfDNA was extracted from fresh plasma samples (within 30 min after drawing) from all patients. cfDNA was isolated from 250 $\mu \mathrm{L}$ of plasma by DNA isolation kit (ArrowDNA Kit; NorDiag, Holliston, MA, USA) using the automatic extractor NorDiag Arrow (NorDiag, Holliston, MA, USA). The DNA obtained was eluted in $50 \mu \mathrm{L}$ of elution buffer. cfDNA extraction was performed in duplicate for each sample.

\section{Real-Time Quantitative PCR}

Plasma cfDNA was measured by real-time quantitative polymerase chain reaction (PCR) using RotorGene 6000 (Qiagen, Milan, Italy). We amplified the $\beta$-actin gene (primers sequences: Forward $\left[5^{\prime}-3^{\prime}\right]$ GCGCCGTTCCGAAAGTT; Reverse [5'-3']: CGGCGGATCGGCAAA).

A volume of $10 \mu \mathrm{L}$ of cfDNA was used as template in each PCR reaction. Each run included an activation step at $95^{\circ} \mathrm{C}$ for $3 \mathrm{~min}$ followed by 50 amplification cycles of $30 \mathrm{~s}$ denaturation, $30 \mathrm{~s}$ annealing at $65^{\circ} \mathrm{C}$ (touchdown $1^{\circ} \mathrm{C}$ for 10 cycles) and 30 s elongation, and a final elongation step at $72^{\circ} \mathrm{C}$ for $5 \mathrm{~min}$, with fluorescence acquired on the green channel. The PCR products were heated to $99^{\circ} \mathrm{C}$ and cooled for heteroduplex formation, and melt was monitored by fluorescence emission using appropriate denaturation range $\left(50-99^{\circ} \mathrm{C}\right)$.

For each run, 1 aliquot of stock DNA was serially diluted to create a 6-point standard calibration curve $(200,000-20,000-2,000-$ $200-20-2 \mathrm{pg} / \mathrm{mL})$. A conversion factor of $6.6 \mathrm{pg}$ of DNA per diploid cell was used. Results are expressed as genome equivalents (GEs)/mL; $1 \mathrm{GE} / \mathrm{mL}$ equals $6.6 \mathrm{pg}$ DNA. We performed PCR runs 2 times in triplicate with samples and standard curves.

\section{Determination of Caspase-3 Activity}

Plasma samples were tested for cell-free caspase- 3 evaluation. Caspase- 3 was measured by Human Caspase-3 Instant ELISA Kit (eBioscience, San Diego, CA, USA) with a fluorometric assay at $450 \mathrm{~nm}$ by VICTORX 4 Multilabel Plate Reader (PerkinElmer Life Sciences, Waltham MA, USA). Each experiment was performed in triplicate. Caspase- 3 concentrations $(\mathrm{ng} / \mathrm{mL})$ were extrapolated from the standard curve.

\section{Cytokines Enzyme-Linked Immunosorbent Assay}

Quantitative determination of tumor necrosis factor- $\alpha$, interleukin (IL)-18, and IL-6 in plasma was performed by Human Instant ELISA kit (eBioscience, San Diego, CA, USA) according to manufacturer's instructions. Optical density was read by using a 
Table 1. Clinical and laboratory parameters of CRS type 1 and AHF patients included in the study

\begin{tabular}{lccc}
\hline & $\begin{array}{c}\text { AHF with CRS type } 1 \\
(n=15)\end{array}$ & $\begin{array}{c}\text { AHF } \\
(n=17)\end{array}$ & $p$ value \\
\hline Age, years & $75.2 \pm 9.0$ & $73.5 \pm 10.2$ & 0.62 \\
Weight, $\mathrm{kg}$ & $80(75-90)$ & $74(65-100)$ & 0.80 \\
Male, \% & 53 & 47 & 0.73 \\
Diabetes, \% & 67 & 76 & 0.55 \\
Hypertension, \% & 87 & 88 & 0.87 \\
Lowest systolic blood pressure, $\mathrm{mm} / \mathrm{Hg}$ & $140(120-157)$ & $150(135-163)$ & 0.76 \\
Lowest diastolic blood pressure, mm/Hg & $80(73-95)$ & $80(75-97)$ & 0.82 \\
Peripheral vascular disease, \% & 40 & 41 & 0.76 \\
Cardiovascular disease, \% & 27 & 29 & 0.87 \\
Obesity, \% & 20 & 24 & 0.82 \\
Dyslipidemia, \% & 33 & 35 & 0.91 \\
Ejection fraction, \% $(n)$ & $35 \%(25-51)$ & $35 \%(24-48)$ & 0.87 \\
SCr at baseline, mg/dL & $1.04(0.92-1.2)$ & $0.94(0.86-1.2)$ & 0.11 \\
SCr at admission, mg/dL & $1.3(1.2-1.4)$ & $0.91(0.83-1.1)$ & $<0.001$ \\
Urea at admission, mg/dL & $774(58-142)$ & $50(41-57)$ & 0.002 \\
Hemoglobin at admission, g/dL & $11.4(9.7-13)$ & $13.6(11.1-14.3)$ & 0.06 \\
Albumin at admission, g/L & $4.2(3.97-4.4)$ & $4.1(3.9-4.3)$ & 0.57 \\
Peak of creatinine, mg/dL & $1.6(1.4-1.7)$ & $0.96(0.91-1.13)$ & 0.04 \\
\hline
\end{tabular}

CRS type I, cardiorenal syndrome type 1; AHF, acute heart failure; SCr, serum creatinine.

VICTORX4 Multilabel Plate Reader (PerkinElmer Life Sciences, Waltham, MA, USA) at $450 \mathrm{~nm}$. The concentration values for these molecules were calculated from standard curves.

\section{Statistical Analysis}

Statistical analysis was performed using the SPSS Software package and GraphPad. A $p$ value of $<0.05$ was considered statistically significant. Categorical variables were expressed as percentages; continuous variables were expressed as mean \pm standard deviation (normally distributed variables) or median and interquartile range (IQR) (non-normally distributed variables). The Mann-Whitney $U$ test or $T$ test was used for comparison of 2 groups when appropriate. Categorical variables were compared using $\chi^{2}$ test. Correlation coefficients were calculated with the Spearman's rank correlation coefficient test and were used to verify the correlation between variables.

\section{Results}

\section{Subjects Baseline Characteristics}

We analyzed the cause of AHF in our population: nonST segment elevation myocardial infarction in $6.3 \%$ of patients, excessive salt and fluid intake in $31.2 \%$ of patients, hypertensive crisis in $18.8 \%$ of patients, and other different reasons in $31.2 \%$ of patients. In $12.5 \%$ of patients, no cause of AHF was recognized.
The mean age of 15 patients with CRS type 1 was 75.2 \pm 9.0 years and $47 \%$ of the patients were females. The median baseline SCr of CRS type 1 patients was $1.04 \mathrm{mg} / \mathrm{dL}$ (IQR 0.92-1.2), with median eGFR $56 \mathrm{~mL} / \mathrm{min} / 1.73 \mathrm{~m}^{2}$ (IQR 48-66). Ten CRS type 1 subjects had diabetes and 13 (87\%) had hypertension. 7/15 CRS type 1 patients developed AKI during in-hospital stay (first $48 \mathrm{~h}$ from admission). The median of length of stay (LOS) was 8 days (IQR 7-11) for CRS type 1 patients. Three CRS type 1 patients died during the in-hospital stay.

The mean age of 17 patients with AHF was $73.5 \pm 10.2$ years and $53 \%$ of these patients were females. The median baseline sCr of AHF subjects was $0.94 \mathrm{mg} / \mathrm{dL}$ (IQR 0.861.2), with median eGFR $66 \mathrm{~mL} / \mathrm{min} / 1.73 \mathrm{~m}^{2}$ (IQR 54-73). Thirteen AHF subjects had diabetes and 15 AHF subjects had hypertension. The median of LOS was 8 days (IQR 6-10) for this group. No patients with AHF died during the study period.

Characteristics of CRS type 1 and AHF patients are described in Table 1. AHF and CRS type 1 patients were similar for age and weight, blood pressure, and ejection fraction and had similar comorbidities. Mortality was significantly higher in CRS type 1 patients $(p=0.05)$. The in-hospital LOS resulted similar in the 2 groups. SCr at baseline and albumin were not significantly different in CRS type 1 and AHF patients $(p=0.11$ and $p=0.57$, re- 


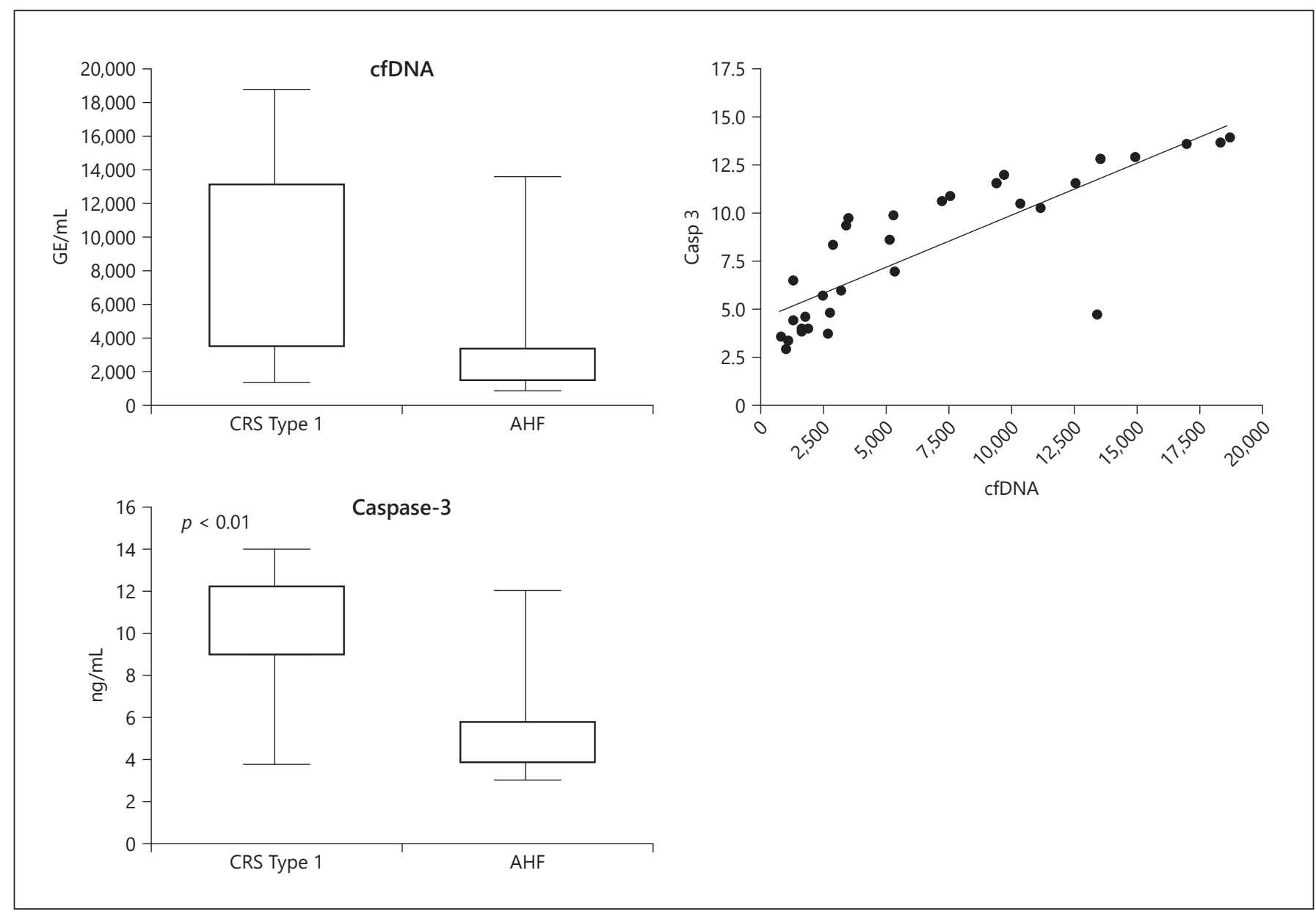

Fig. 1. Plasma cfDNA and caspase-3 levels in patients with CRS type 1 and AHF. cfDNA, cell-free DNA; CRS type I, cardiorenal syndrome type 1; AHF, acute heart failure; Casp 3, caspase-3; GEs, genome equivalents.

spectively). Moreover, there was no significant difference in terms of median hemoglobin among AHF and CRS type 1 patients $(p=0.06)$. However, there was a tendency toward lower level of hemoglobin in CRS type 1 patients (11.4 g/dL, IQR 9.7-13.0 vs. 13.6 g/dL, IQR 11.1-14.3). At admission, SCr and urea levels resulted significantly higher in CRS type 1 patients than AHF subjects $(p<$ 0.001 and $p=0.02$, respectively). The median variation of $\mathrm{SCr}$ was significantly higher in CRS type 1 group (0.35 $\mathrm{mg} / \mathrm{dL}$, IQR $0.30-0.51)$ respect AHF patients $(0.08 \mathrm{mg} /$ dL, IQR 0.04-0.12) $(p=0.05)$.

\section{cfDNA Levels in AHF and CRS Type 1 Patients}

We observed significantly higher levels of cfDNA in patients with CRS type $1(7,531 \mathrm{GE} / \mathrm{mL}$; IQR: 3,46013,060 vs. $1,921 \mathrm{GE} / \mathrm{mL}$; IQR: $1,623-3,185$ in AHF pa- tients, $p<0.01$ ) (Fig. 1). No significant differences were found between the levels of cfDNA in patients who were admitted with AKI and patients who developed AKI during in-hospital stay (7,371 GE/mL; IQR: 3,921-12,017 vs. 5,331 GE/mL; IQR: 2,087-10,272, $p=0.40$ ).

$3 / 15$ CRS type 1 patients died during the in-hospital stay. The cfDNA concentrations at admission were similar in CRS type 1 survivors and nonsurvivors $(8,463 \mathrm{GE} /$ mL; IQR: 3,487-13,894 vs. 7,211 GE/mL; IQR: 4,958$8,781)(p=0.4)$.

\section{Plasma Caspase-3 Levels in AHF and CRS Type 1 Patients}

The median value of plasma caspase- 3 was $4.6 \mathrm{ng} / \mathrm{mL}$ (IQR 3.8-5.6) in AHF patients. The median level of plasma caspase- 3 was $10.5 \mathrm{ng} / \mathrm{mL}$ (IQR 9.0-12.2) in CRS type 
Table 2. NGAL levels and cytokine profile

a NGAL levels and cytokine profile in CRS type 1 and AHF patients included in the study

\begin{tabular}{lccc}
\hline & AHF with CRS type 1 & AHF & $p$ value \\
\hline NGAL, ng/mL & $291(213-1,300)$ & $197(144-239)$ & 0.04 \\
TNF- $\alpha, \mathrm{pg} / \mathrm{mL}$ & $32.2(26.9-38.3)$ & $32.8(29.8-37.0)$ & 0.90 \\
IL-6, pg/mL & $86.9(76.6-98.6)$ & $23.2(18.9-24.3)$ & $<0.001$ \\
IL-18, pg/mL & $107(88.7-150.2)$ & $77.8(71.8-89.5)$ & 0.02 \\
\hline
\end{tabular}

b NGAL and cytokine levels in CRS type 1 patients who were admitted with AKI and patients who developed AKI during in-hospital stay

\begin{tabular}{lccc}
\hline & AKI at admission & AKI during in-hospital stay & $p$ value \\
\hline NGAL, ng/mL & $391(207-1,147)$ & $256(187-925)$ & 0.82 \\
TNF- $\alpha, \mathrm{pg} / \mathrm{mL}$ & $32.9(26.7-39.3)$ & $31.9(28.7-35.3)$ & 0.90 \\
IL-6, pg/mL & $93.7(83.5-107.8)$ & $79.0(71.9-88.0)$ & 0.54 \\
IL-18, pg/mL & $101.2(88.3-291.3)$ & $116.2(94.4-120.3)$ & 0.15 \\
\hline
\end{tabular}

c NGAL and cytokine levels at admission in CRS type 1 survivors and nonsurvivors

\begin{tabular}{lccc}
\hline & CRS type 1 survivors & CRS type 1 nonsurvivors & $p$ value \\
\hline NGAL, ng/mL & $289(200.2-1,146.8)$ & $256(227-373.5)$ & 0.12 \\
TNF- $\alpha, \mathrm{pg} / \mathrm{mL}$ & $31.9(26.7-38.1)$ & $32.9(30.2-36.9)$ & 0.96 \\
IL-6, pg/mL & $88.0(74.3-111.3)$ & $90.7(88.8-93.8)$ & 0.49 \\
IL-18, pg/mL & $107.5(89.2-141.2)$ & $95.6(83.8-135.8)$ & 0.30 \\
\hline
\end{tabular}

CRS type I, cardiorenal syndrome type 1; AHF, acute heart failure; AKI, acute kidney injury; TNF- $\alpha$, tumor necrosis factor- $\alpha$; IL, interleukin.

1 patients. The level of caspase- 3 resulted in significantly increase in CRS type 1 patients $(p<0.01)$ (Fig. 1).

No significant differences were found between the levels of plasma caspase- 3 in patients who were admitted with AKI and patients who developed AKI during in-hospital stay ( $10.5 \mathrm{ng} / \mathrm{mL}$; IQR: 7.5-11.4 vs. $9.9 \mathrm{ng} / \mathrm{mL}$; IQR: $7.4-10.9, p=040$ ). The plasma caspase-3 at admission were similar in CRS type 1 survivors and nonsurvivors (10.7 ng/mL; IQR: $9.7-12.8$ vs. $8.4 \mathrm{ng} / \mathrm{mL}$; IQR: $6.1-9.5)$ ( $p=0.3)$.

\section{Cytokines Profile}

Cytokines profile is described in Table 2. Tumor necrosis factor- $\alpha$ levels in plasma were similar in AHF and CRS type 1 patients $(p=0.86)$. Furthermore, plasmatic pro-inflammatory cytokines (IL-6 and IL-18) were significantly higher in CRS type 1 patients $(p<0.001$ and $p=0.024$, respectively). Furthermore, NGAL levels re- sulted significantly higher at admission in CRS type 1 patients than AHF patients (291 ng/mL, IQR 213-1,300 vs. $197 \mathrm{ng} / \mathrm{mL}$, IQR 144-239, $p=0.02$ ).

No significant differences were found between the levels of NGAL and cytokines in patients who were admitted with AKI and patients who developed AKI during in-hospital stay (Table 2). The levels of inflammatory molecules at admission were similar in CRS type 1 survivors and nonsurvivors (Table 2).

\section{Correlation}

We observed a positive strong correlation between cfDNA and caspase-3 levels (Spearman's rho $=0.895, p<$ 0.001 ) and between cfDNA and IL-6 (Spearman's rho $=$ $0.728, p<0.001$ ) (Fig. 2). Furthermore, we observed positive correlation between cfDNA levels and renal parameters (Fig. 2). 


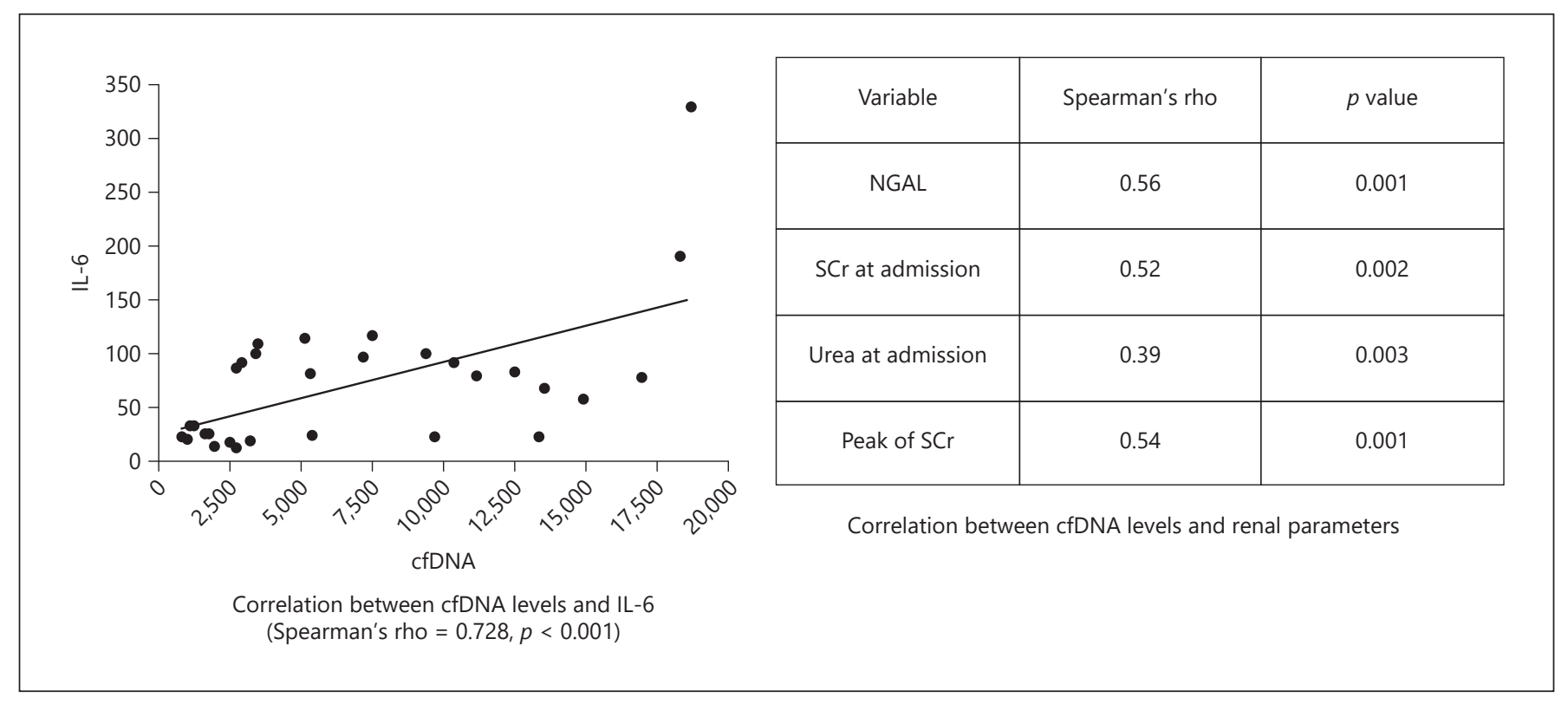

Fig. 2. Correlation between cfDNA levels and IL- 6 and cfDNA and renal parameters. cfDNA, cell-free DNA; SCr, serum creatinine.

\section{Discussion}

The biological role and the levels of cfDNA in patients with AHF and patients with CRS type 1, and its association with caspase-3 levels, inflammatory, and renal parameters have not been yet explored.

cfDNA was first described by Mandel and Metais [15] in 1948 as nucleic acids in peripheral blood deriving from cell death induced by injury, apoptosis, or cell necrosis. Different cellular lines (i.e., neutrophils, eosinophils, macrophages, etc.) may release cfDNA, particularly in specific clinical conditions, such as sepsis, trauma, myocardial infarction, etc. [1-9]. Most of cfDNA circulates in the form of mononucleosomes, which protect it from degradation; hence, internucleosomic degradation of chromatin, typical of apoptosis, was suggested as a potential source of cfDNA [16]. In higher organisms, apoptosis is intermediated by caspases, a family of cysteine proteases cleaving after an aspartate residue in their substrates [11].

In our study, cfDNA levels were higher in patients with CRS type 1 than patients with AHF. Even though the 2 groups have similar epidemiological characteristics and comorbidities, data on NYHA classes are not available, and this limitation should be considered. In fact, the severity of heart failure could affect cfDNA levels. In CRS type 1 cohort, no significant difference was found in terms of cfDNA levels between patients admitted with $\mathrm{AKI}$ and patients who developed AKI during first $48 \mathrm{~h}$ of in-hospital stay.

CRS type 1 is distinguished by a rapid deteriorating of cardiac function, which leads to AKI. Low cardiac output is responsible for the reduced kidney perfusion and the resultant renal ischemia with AKI. Also nonhemodynamic mechanisms, such as inflammation and immune dysregulation, participate in the pathogenesis of CRS type 1. Additionally, apoptosis of epithelial cells in proximal and distal tubules has an important role in ischemic renal injury [10].

The higher levels of cfDNA in patients with CRS type 1 than patients with AHF may derive from apoptotic pathways activated in the setting of AKI complicating AHF. According to our data, caspase-3 levels were higher in patients with CRS type 1 . In fact, a positive correlation between cfDNA and caspase-3 levels was highlighted. We can hypothesize that in patients with CRS type 1, caspase-3 levels are increased due to the activation of apoptotic mechanisms responsible for AKI, and they correlate with cfDNA concentrations. No significant difference was found in terms of caspase-3 levels between patients admitted with AKI and patients who developed AKI during in-hospital stay. These results could be related to the physiological timing of apoptosis: apoptosis occurs 6-12 $\mathrm{h}$ after the kidney insult [10]. 
Based on the previous studies, we hypothesized that cfDNA concentrations derived from pathogenic apoptosis reported in monocytes and in renal tubular epithelial cells in CRS type 1 [17-20]. Apototsis is highly regulated process. It is an essential physiological mechanism, but it can generate havoc if stimulated inappropriately, creating a deleterious cross-talk. Changes at the cellular level are fundamental for detrimental tissue remodeling and anomalous bidirectional link between heart and kidney [21]. Recently, Savira et al. [22] reported that the inhibition of apoptosis signal-regulating kinase 1 ameliorates left ventricular dysfunction, reduces hypertrophy and fibrosis in a rat model of CRS. In this context, the use of cfDNA, as a biomarker, could be a good, noninvasive and indirect indicator of apoptosis in CRS type 1 patients. Furthermore, cfDNA could be a possible method for evaluating and monitoring new therapeutic approaches for these patients.

Furthermore, in our study, IL-6 and IL-18 levels were higher in patients with CRS type 1. These data confirmed the inflammatory mechanisms and immune dysregulation implicated in the pathogenesis of CRS type $1[17,18]$. In the context of cfDNA, previous study demonstrated, cfDNA induces IL-6 production in human monocytes in hemodialysis patients [23]. Future studies are needed to verify this causale connection between cfDNA and IL- 6 in the setting of CRS type 1. In addition, cfDNA levels have been found to correlate with renal parameters as well, and this may be due to the extent of renal damage: as greater the kidney damage, as greater cfDNA concentrations. The mechanism by which cfDNA is removed from the body and its renal clearance is not known. Therefore, we cannot state if the increase in cfDNA levels is due to a reduced renal removal in the setting of AKI or to its highly dysregulated production.

Pro-inflammatory cytokines provoke other immune cells to produce additional inflammatory markers, creating an environment for cell death and altering organ fate. In the context of CRS, Pastori et al. [17] justify the notion that inflammation alters organ destiny compared to other compensatory mechanisms in an in vitro experimental study. Moreover, recent evidence shows that patients with severe heart failure and AKI demonstrate high proinflammatory cytokines, chemokine upregulation, neutrophil migration and extravasation, toll-like receptor expression, and unregulated apoptosis leading to oxidative stress $[24,25]$.

Unfortunately, we cannot establish a causal relationship between cfDNA levels, inflammatory markers, and renal parameters, but we can hypothesize a possible association. Unfortunately, the design of this pilot study does not allow us to make conclusions about causality. Further studies are needed to evaluate the casual relationship and the effect of the severity of heart failure on $\mathrm{cfD}$ NA levels.

cfDNA has been found in many pathological settings in which apoptosis is involved [23, 26]. Our study explores the premise of cfDNA as a marker for apoptosis and monitoring inflammatory state in the setting of CRS type 1.

cfDNA could potentially serve as an index for noninvasive monitoring of tissue damage and apoptosis in patients with AKI induced by AHF. However, the exact characteristics of cfDNA kinetics and clearance remained uncertain. Furthermore, potential functional effects of cfDNA have not been yet investigated. Further future studies are required to explain the precise mechanism and the clinical significance of elevated cfDNA in this population. These data obtained from this small hypothesis-generating pilot study provide the basis for larger studies that may clarify the efficacy and the utility of cfDNA in CRS type 1 patients and the contribution of inflammation in this population.

\section{Acknowledgments}

The authors thank nurses of the Internal Medicine Department of San Bortolo Hospital.

\section{Statement of Ethics}

All procedures were in accordance with the Helsinki Declaration. The protocol and consent form were approved by the Ethics Committee of San Bortolo Hospital (Sperimentazione No. 69/09). All patients were informed about the experimental protocol, and the objectives of the study, and they all gave written informed consent. None of the patients denied the written consent. This statement is to certify that all authors have seen and approved the manuscript being submitted. We warrant that the article is the authors' original work. We warrant that the article has not received prior publication and is not under consideration for publication elsewhere. On behalf of all co-authors, the corresponding author shall bear full responsibility for the submission. This research has not been submitted for publication nor has it been published in whole or in part elsewhere. We attest to the fact that all authors listed on the title page have contributed significantly to the work, have read the manuscript, attest to the validity and legitimacy of the data and its interpretation, and agree to its submission. All authors agree that author list is correct in its content and order and that no modification to the author list can be made without the formal approval of the Editor-in-Chief. No additional authors will be added post 
submission, unless editors receive agreement from all authors and detailed information is supplied as to why the author list should be amended.

\section{Conflict of Interest Statement}

In the last 3 years, C.R. has been consulting or part of advisory boards for ASAHI, Astute, Baxter, Biomerieux, B. Braun, Cytosorbents, ESTOR, FMC, GE, Jafron, Medtronic, and Toray. The other authors have no conflicts of interest to declare.

\section{Funding Sources}

This work was supported by research grants from the Veneto Region (RF-VEN303/09)

\section{Author Contributions}

G.M.V.contributed to conception of the study, interpretation of data, statistical analysis, management of patients, laboratory analysis, and drafting the article, A.C. contributed to interpretation of data and drafting the article, C.C. contributed to conception of the study and interpretation of data, and laboratory analysis, A.A. and G.G. contributed to conception of the study and final approval of the draft, G.V. and C.C.: providing intellectual content of critical importance to this work. All the authors approved the final version of this article.

\section{Data Availability Statement}

All data generated or analyzed during this study are included in this article. Further inquiries can be directed to the corresponding author.

\section{References}

1 Avriel A, Paryente Wiessman M, Almog Y, Perl Y, Novack V, Galante O, et al. Admission cell free DNA levels predict 28-day mortality in patients with severe sepsis in intensive care. PloS One. 2014;9(6):e100514.

2 Garnacho-Montero J, Huici-Moreno MJ, Gutiérrez-Pizarraya A, López I, MárquezVácaro JA, Macher H, et al. Prognostic and diagnostic value of eosinopenia, C-reactive protein, procalcitonin, and circulating cell-free DNA in critically ill patients admitted with suspicion of sepsis. Crit Care. 2014;18(3):R116.

3 Clementi A, Virzì GM, Brocca A, Pastori S, de Cal M, Marcante S, et al. The Role of cell-free plasma DNA in critically ill patients with sepsis. Blood Purif. 2016;41(1-3):34-40.

4 Shoham Y, Krieger Y, Perry ZH, Shaked G, Bogdanov-Berezovsky A, Silberstein E, et al. Admission cell free DNA as a prognostic factor in burns: quantification by use of a direct rapid fluorometric technique. Biomed Res Int. 2014;2014:306580.

5 Chang CP, Chia RH, Wu TL, Tsao KC, Sun $\mathrm{CF}, \mathrm{Wu}$ JT. Elevated cell-free serum DNA detected in patients with myocardial infarction. Clin Chim Acta. 2003;327(1-2):95-101.

6 Rainer TH, Wong LK, Lam W, Yuen E, Lam NY, Metreweli C, et al. Prognostic use of circulating plasma nucleic acid concentrations in patients with acute stroke. Clin Chem. 2003;49(4):562-9.

7 Taglauer ES, Wilkins-Haug L, Bianchi DW. Review: cell-free fetal DNA in the maternal circulation as an indication of placental health and disease. Placenta. 2014;35 (Suppl 1):S64-8.

8 Korabecna M, Opatrna S, Wirth J, Rulcova K, Eiselt J, Sefrna F, et al. Cell-free plasma DNA during peritoneal dialysis and hemodialysis and in patients with chronic kidney disease. Ann NY Acad Sci. 2008;1137:296-301.

9 Kim K, Shin DG, Park MK, Baik SH, Kim TH, Kim S, et al. Circulating cell-free DNA as a promising biomarker in patients with gastric cancer: diagnostic validity and significant reduction of cfDNA after surgical resection. Ann Surg Treat Res. 2014;86(3):136-42.

10 Devarajan P. Update on mechanisms of ischemic acute kidney injury. J Am Soc Nephrol. 2006;17(6):1503-20.

11 Fuentes-Prior P, Salvesen GS. The protein structures that shape caspase activity, specificity, activation and inhibition. Biochem J. 2004;384(Pt 2):201-32.

12 McMurray JJ, Adamopoulos S, Anker SD, Auricchio A, Böhm M, Dickstein K, et al. ESC guidelines for the diagnosis and treatment of acute and chronic heart failure 2012: the task force for the diagnosis and treatment of acute and chronic heart failure 2012 of the European society of cardiology. Developed in collaboration with the heart failure association (HFA) of the ESC. Eur J Heart Fail. 2012; 14(8):803-69.

13 Mehta RL, Kellum JA, Shah SV, Molitoris BA, Ronco C, Warnock DG, et al. Acute kidney injury network: report of an initiative to improve outcomes in acute kidney injury. Crit Care. 2007;11(2):R31.

14 Levey AS, Stevens LA, Schmid CH, Zhang YL, Castro AF 3rd, Feldman HI, et al. A new equation to estimate glomerular filtration rate. Ann Intern Med. 2009;150(9):604-12.

15 Mandel P, Metais P. Nuclear acids in human blood plasma. C R Seances Soc Biol Fil. 1948; 142(3-4):241-3.

16 Lichtenstein AV, Melkonyan HS, Tomei LD, Umansky SR. Circulating nucleic acids and apoptosis. Ann NY Acad Sci. 2001;945:239-49.

17 Pastori S, Virzì GM, Brocca A, de Cal M, Clementi A, Vescovo G, et al. Cardiorenal syndrome type 1: a defective regulation of monocyte apoptosis induced by proinflammatory and proapoptotic factors. Cardiorenal Med. 2015;5(2):105-15.
18 Virzi GM, de Cal M, Day S, Brocca A, Cruz DN, Castellani C, et al. Pro-apoptotic effects of plasma from patients with cardiorenal syndrome on human tubular cells. Am J Nephrol. 2015;41(6):474-84.

19 Pastori S, Virzì GM, Brocca A, de Cal M, Cantaluppi V, Castellani C, et al. Cardiorenal syndrome type 1: activation of dual apoptotic pathways. Cardiorenal Med. 2015;5(4):30615.

20 Virzi GM, Clementi A, Ronco C. Cellular apoptosis in the cardiorenal axis. Heart Fail Rev. 2016;21(2):177-89.

21 Peesapati VSR, Sadik M, Verma S, Attallah MA, Khan S. Panoramic dominance of the immune system in cardiorenal syndrome type I. Cureus. 2020;12(8):e9869.

22 Savira F, Wang BH, Edgley AJ, Jucker BM, Willette $\mathrm{RN}$, Krum $\mathrm{H}$, et al. Inhibition of apoptosis signal-regulating kinase 1 ameliorates left ventricular dysfunction by reducing hypertrophy and fibrosis in a rat model of cardiorenal syndrome. Int J Cardiol. 2020;310: 128-36.

23 Atamaniuk J, Kopecky C, Skoupy S, Säemann MD, Weichhart T. Apoptotic cell-free DNA promotes inflammation in haemodialysis patients. Nephrol Dial Transplant. 2012;27(3): 902-5.

24 Li X, Hassoun HT, Santora R, Rabb H. Organ crosstalk: the role of the kidney. Curr Opin Crit Care. 2009;15(6):481-7.

25 Ronco C, Cicoira M, McCullough PA. Cardiorenal syndrome type 1: pathophysiological crosstalk leading to combined heart and kidney dysfunction in the setting of acutely decompensated heart failure. J Am Coll Cardiol. 2012;60(12):1031-42.

26 Jung K, Fleischhacker M, Rabien A. Cell-free DNA in the blood as a solid tumor biomarker: a critical appraisal of the literature. Clin Chim Acta. 2010;411(21-22):1611-24. 